\title{
Ultra Wide Band Body Area Networks: Design and Integration with Computational Clouds
}

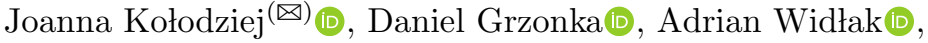 \\ and Paweł Kisielewicz (D) \\ Department of Computer Science, Cracow University of Technology, \\ ul. Warszawska 24, 31-115 Cracow, Poland \\ jokolodziej@pk.edu.pl
}

\begin{abstract}
Body Area Networks (BANs) connect together nodes attached to a human body and transfer the data to an external infrastructure. The wireless communication channel and a variety of miniature sensor devices have lead to many useful applications of BANs, such as healthcare monitoring, military and emergency coordination, rescue services, sports, and entertainment. The Ultra Wide Band (UWB) communication model is widely used in wireless body area networks. UWB Radio Frequency (RF) technology provides robust and energy efficient transmission of data and signals through wireless networks. This chapter surveys recent models, applications and research challenges for future generation UWB RF technology for BANs. The chapter also discusses the state-of-the art in the cloud-based support for data storage and analysis in mobile health monitoring. Security issues for BANs in general and mobile health monitoring are addressed as a key aspect of the recent developments in the domain.
\end{abstract}

Keywords: Cloud computing - Body area networks •

Sensor networks • Ultra wideband communication · Medical services •

Wireless communications

\section{Introduction}

Based on the IEEE 802.15 standards, Body Area Network (BAN) can be defined as "a communication standard optimized for low power devices and operation on, in or around the human body (but not limited to humans) to serve a variety of applications including medical, consumer electronics/personal entertainment and others [5]". REcently, technological advancements in ultra low-power Radio Frequency (RF) technology, low-power integrated circuits, energy harvesting and storage, and wireless communications have lead to the design of lightweight, intelligent, and low-cost medical devices and sensors. With all these achievements, the thought of widespread deployment of pervasive wireless BANs for diverse 
applications, such as health monitoring and military coordination, seems to be a reality of the near future [27].

Typical BAN connects self-regulating nodes (sensors, artificial skin, etc.) attached to the surface of body or implanted in the body [85]. The focus in BANs has been on medical and quality of life applications [70]. In such applications the network's nodes can be implanted medical devices, such as electrocardiogram (ECG) electrodes, activity sensors and actuators, or devices for storage of the medical data [70]. The Wireless Body Area Network (WBAN) sensors and devices may be classified based on their location into the following two main categories [3]:

- in-body nodes,

- on-body area network nodes.

Figure 1 presents the generic model of typical WBAN in-body and on-body nodes distribution with the wrist node as a local (central) base station.

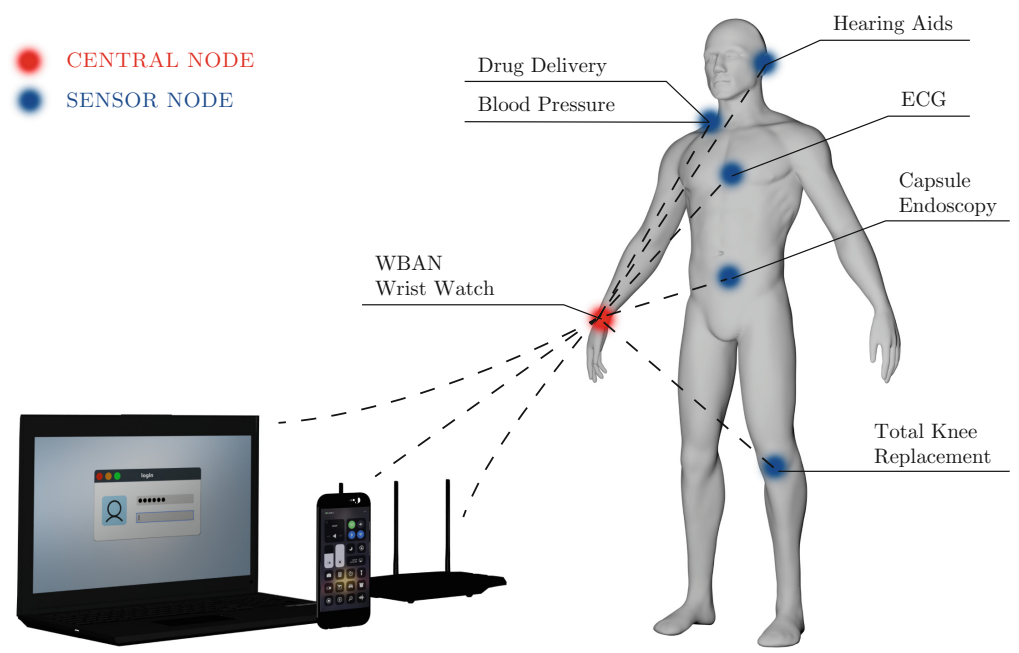

Fig. 1. Generic sample model of wireless BAN [18]

The key requirements for BANs are: (i) small network scale, (ii) long battery life, (iii) short range communications and (iv) security awareness [32,85]. Many powerful sensor platforms have been developed for various applications in the past decade [75]. In the case of transceivers, decreasing the amount of energy required to transmit a bit of data means: (a) reducing the turn on/off times and power during transients, and (b) duty cycling the radio operation [41].

The Ultra Wide Band (UWB) is the key technology for data transmission in WBANs through the low-power transmission and low-power duty cycle [47]. In Ultra Wide Band Body Area Networks (UWB-BANs), the human tissues are not affected by closely placed WBAN devices. Low-power transmission and 
duty cycle also enable lower power consumption in the network nodes and long battery time, which is the basic requirement for such systems. UWB technology also conforms to the size and cost constraints of BAN devices [27]. From the implementation perspective, the major advantages of using UWB radios are: (a) low implementation complexity, (b) low duty cycling operation, and (c) bit rate scalability [22].

UWB BANs have been surveyed already from the different aspects $[12,47]$ such as communication channel models $[8,72,73,81]$, transmitter design $[34,70]$, antenna design [38,72], and performance evaluation [13,23,73]. This chapter summarizes all the above surveys and gives the general and wider view on the design, architecture and technological issues. It addresses the main challenges related to BANs and UWB-BANs and presents the recently developed models based on the cloud computing support for the patient's health monitoring, patients' data storage, analysis and processing with the security criterion for inter and intra-cloud communication and data access.

The rest of the chapter is organized as follows. BANs application areas are discussed in Sect.2.1. Section 2.2 presents the classification of the wireless technologies used in WBANs. In Sect. 2.3, some practical issues and relations between different requirements of BANs are discussed. Sections 2.4 and 3 define the research challenges and state-of-the art in UWB BANs. The concept of cloud-based support for WBANs is presented in Sect. 4. Security aspects related to the cloud support in mobile health applications based on UWB-BANs are discussed in Sect. 5. The chapter ends with the conclusions and highlights of the future research directions in the domain specified in Sect. 6.

\section{BAN's Applications and Challenges}

Various types of the main BANs components, namely, vital sign monitoring sensors, motion detectors (through accelerometers) and communication protocols for data processing and system access, make these networks very useful in providing numerous services in healthcare, research, lifestyle, emergency, sports, and military $[12,26,32]$. In this section we first discuss the major application areas for BANs, present the candidate technologies for wireless communication and data transmission, and then highlight the most important practical and research challenges regarding WBAN technologies.

\subsection{Applications of BANs}

The major areas of applications of BANs can be specified as follows.

Healthcare: BANs are used to connect sensor devices with remote monitoring and assistance systems for physically disabled and elderly persons. Mobile devices can be used to send and receive data to hospitals from the ambulances carrying patients to alert the concerned authorities and to get information about providing first aid to save people's lives [32]. BANs also enable a continuous monitoring of patient's condition by sensing and transmitting vital signs, such as 
heart rate, ECG, body temperature, respiratory rate, chest sounds, blood pressure, etc. $[9,41]$. It makes BANs an important tool for diagnosis and treatment of patients with chronic diseases, such as hypertension, diabetes, etc. BANs are also beneficial to hospital patients who are monitored pervasively regardless of their location. Pervasive in-patient monitoring through implanted devices enables medical staff to predict, diagnose, and start treatment before the patient reaches an adverse stage of the disease [26,77]. BANs are also highly beneficial for monitoring and assistance of elderly people, as more and more people demand a better quality of life.

Work and Emergency Services: BANs are used in emergency services like rescue systems, firefighting, etc. [47]. Using miniature sensors, the condition and location of firefighters is monitored. These sensors are also used to transfer information to a person, e.g., a commander who is not present at the site. Based on the information provided by sensors, the commander can coordinate emergencies with the team carrying out the rescue operation.

Lifestyle and Sports: BANs are also beneficial in providing quality of life and sport healthcare monitoring [83]. Such applications may include wearable entertainment systems, car navigation systems, and tour guides. In sports, BANs are used to monitor a player vital signs, such as the heart rate of a marathon runner.

Military: BANs are also used in war and combat scenarios by the military and police personnel. Wearable sensors are integrated into the uniforms of soldiers. This network can then be used to connect different devices, such as health sensors, surveillance cameras, and Personal Digital Assistants (PDAs) installed on different personnel body parts [16].

It is envisioned that wireless body area networks will become the heart of the future Internet and will play a vital role in access and exchange of information in order to provide better facilities in healthcare, education, and lifestyle [41]. Realtime patients vital signs monitoring, emergency and battlefield coordination may be read as a part of science fiction and movies. But one such ambitious project is underway at University of Washington, to display real-time information gathered from different body sensors through LEDs built in contact lenses [58].

\subsection{Candidate Wireless Technologies}

In this section, various wireless technologies that are leading competitors in the upcoming market of BANs are discussed. Communication between sensor devices is called intra-BAN communication while BAN communication with other networks is called extra-BAN communication [37]. Selection of appropriate technology for intra-BAN communication is a research issue where end-to-end performance of candidate wireless technologies is determined by the complete protocol stack (i.e., including Physical layer (PHY) and upper protocol layers).

Bluetooth Classic: Bluetooth is a short range wireless communication standard that defines the link and application layers to support data and voice 
applications. Piconet is a short range network made up of up to eight Bluetooth devices. Bluetooth Special Interest Group has developed the Bluetooth Health Device Profile (HDP) that defines the requirements for qualified Bluetooth healthcare and fitness device implementations. Bluetooth is widely adopted wireless technology for intra-BAN communication in WBANs [66].

Bluetooth Low Energy: Bluetooth Low Energy (BTLE) is another candidate wireless technology for BANs. It provides simple device discovery and reliable point-to-multipoint data transfer, with encryption, power save functionalities, and ultra low power idle mode operation. The key advantages of BTLE are the strength of the Bluetooth brand, the promise of interoperability with Bluetooth radios in mobile phones and low-power operations [66].

ZigBee: ZigBee defines a network, security, and application layer protocol suite on top of the PHY and Media Access Control (MAC) layers defined by the IEEE 802.15.4 Wireless Personal Area Network (WPAN) standard. The PHY layer exploits the direct sequence spread spectrum technique for interference tolerance and MAC layer exploits carrier sense multiple access with collision avoidance (CSMA/CA) for channel access. ZigBee also provides support for personal health devices complying with the IEEE 11073 standard, including electrocardiographs, glucometers, pulse oximeters, blood pressure monitors, thermometers, weight scales, and respirometers [12].

ANT: ANT is a proprietary technology designed for general purpose wireless personal area network applications. ANT features low latency, simple design, ability to trade off a data rate against power consumption, and a net data rate of $20 \mathrm{~kb} / \mathrm{s}$ (over-the-air data rate is $1 \mathrm{Mb} / \mathrm{s}$ ) [12].

Sensium: Sensium is a proprietary ultra-low-power transceiver platform custom designed for healthcare and lifestyle management applications. The network adopts a master-slave architecture where joining and leaving the network is managed centrally and all communications are single-hop [12].

Zarlink: Zarlink has developed an ultra-low-power RF transceiver, ZL70101, for medical implantable applications. It uses a Reed-Solomon coding scheme together with cyclic redundancy check (CRC) in order to achieve a highly reliable link. The key features of Zarlink ZL70101 are extremely low power consumption, ultra-low power wakeup circuit, MedRadio compliance and security [12].

Other Technologies: Proprietary RF technologies such as BodyLAN and ZWave are also emerging on the horizon. Inductive coupling (IC) and body coupled communications (BCC) technologies are also promising. The data rate of IC is limited, and it cannot initiate communication from inside the body. BCC transceivers are capacitively coupled to the skin and use the human body as a channel to exchange data. BCC is energy efficient, and alleviates interference and coexistence issues. BCC can also be used for user identification and automatic formation of BANs [66]. 


\subsection{Practical Challenges}

BANs face several important challenging issues regarding their efficiency, practical deployment, and social adoption $[32,54]$. These issues constrain the solution space, and need to be considered carefully when designing mechanisms for data security and privacy in WBANs.

Conflict Between Security and Efficiency: High efficiency is strongly demanded for data security in WBANs because of the resource constraints. Wearable sensors are often extremely small and have insufficient power supplies, which render them inferior in computation and storage capabilities. Thus, the cryptographic primitives used by the sensor nodes should be as lightweight as possible, in terms of both fast computation and low storage overhead [41].

Conflict Between Security and Safety: Whether the data can be obtained whenever needed or not can be very crucial for patients' safety. Too strict and inflexible data access control may prevent the medical information from being accessed on time by legitimate medical staff, especially in emergency scenarios where the patient may be unconscious and unable to respond. On the other hand, a loose access control scheme opens back doors to malintent users. It is hard to ensure strong data security and privacy while allowing flexible access [20].

Conflict Between Security and Usability: The devices should be easy to use and foolproof, since the users might be non-expert patients. As the setup process of the data security procedures is patient-related, it should involve as few interactions with humans as possible. For instance, to bootstrap initial secure communication between all the nodes in a WBAN for secure data communication, device pairing techniques can be adopted [20]. The devices should offer security services during the phase of data collecting and processing by the advanced data processing centers like, Cloud or Big Data processing systems [78], but not disturbing their daily usage.

Requirement for Device Interoperability: Patients can buy sensor devices from different manufacturers, it may be difficult to pre-share any cryptographic materials among them. It would be hardly possible to establish data security procedures that require minimum settings and efforts, and can work with a wide range of devices [54].

\subsection{Research Challenges}

In this section we summarize different research challenges associated with BANs $[12,26,66]$. These issues need to be addressed for the widespread deployment of BANs. BANs brings forward a number of research issues that need to be considered in the design of RF wireless systems. Users can carry several BAN devices globally, hence, BAN radios are required to operate worldwide. There is an abundance of high power technologies in Industrial, Scientific, and Medical radio bands (ISM bands) that can cast performance degradation on the low-power BAN devices which thus makes them less appealing for high fidelity 
medical applications. Wireless Medical Telemetry Service (WMTS) bands are heavily used but their use is restricted to healthcare facilities in the United States. UWB can be exploited for wearable applications but it raises the issue of coexistence with high-datarate multimedia applications [12]. The rules for MedRadio wing band are very strict and limiting [21]. These issues have provoked the Federal Communication Commission (FCC) to think about opening up 2360-2400 MHz range for medical BANs. This is planned to hold up wideband entrenched micro-stimulator devices that can serve as an artificial nervous system to reinstate sensation, mobility, and function to paralyzed limbs and organs [66].

Another research issue in BANs is a channel model design. The channel model plays a vital role in the design of PHY technologies. Experimental channel modeling for embedded and wearable devices is difficult because humans and healthcare facilities are involved and both are governed by regulations [72].

An antenna design for body area networks is yet another challenging issue due to limitations on the size, stuff, and form of the antenna [84]. Only noncaustic and biocompatible material, such as titanium or platinum, can be used for implants, which results in degraded performance when compared with the antennas made of copper. Organ and location of antenna decides its shape and size which further restricts the choice of designer $[66,82]$.

A physical layer protocol design requires reducing power consumption without affecting reliability. Flawless connectivity should be maintained in dynamic environments without the slightest possible performance degradation in terms of throughput, data loss, and latency. Rapid turnaround time from transmission to reception and speedy wakeup from sleep mode can add significance to power savings [26].

Energy efficient hardware is also an issue; existing wireless technologies draw relatively high peak current and mainly rely on duty cycling the radio between sleep and active modes to minimize the average current drawn. Researchers have been exploring several promising techniques, such as low-power listening and wake-up radios, which are intended to minimize power consumed by idle listening [47].

Coexistence of multiple BANs in crowded places, such as hospitals, needs a robust MAC protocol. The MAC protocol should be able to cope with topology changes caused by movement of nodes. Channel migration protocols need to be developed to be able to migrate to a quiet channel when serious hindrance is noticed [26].

Medical devices are subject to strict regulations to promote the safety and welfare of users. Compliance to applicable regulations set forth by the FCC, U.S. Food and Drug Administration (FDA), European Telecommunications Standards Institute (ETSI), and other regulatory agencies is essential [66].

\section{UWB Solutions for BANs}

Communication and efficient data transmission are the crucial issues in the design and management of modern WBANs. The Federal Communication Commission urges the use of WMTS for medical applications. However, the people 
Table 1. Main properties of communication and data transmission bands for BANs and WBANs [66].

\begin{tabular}{|c|c|c|c|}
\hline \multirow[t]{2}{*}{ Frequency $(\mathrm{MHz})$} & \multirow[t]{2}{*}{ Acronym } & \multicolumn{2}{|c|}{ Scalability to BAN applications } \\
\hline & & Merits & Demerits \\
\hline $401-406$ & MedRadio & $\begin{array}{l}\text { Worldwide availability, } \\
\text { good propagation } \\
\text { characteristics, quiet } \\
\text { channel, medical only }\end{array}$ & $\begin{array}{l}\text { Secondary usage, } \\
\text { body-worn applications } \\
\text { not allowed in } \\
402-405 \mathrm{MHz} \text { core } \\
\text { band, large antenna } \\
\text { size, limited } \\
\text { bandwidth, stringent } \\
\text { rules }\end{array}$ \\
\hline $433.05-434.79$ & $\begin{array}{l}\text { General } \\
\text { Telemetry }\end{array}$ & $\begin{array}{l}\text { Good propagation } \\
\text { characteristics }\end{array}$ & $\begin{array}{l}\text { Not internationally } \\
\text { agreed, } \\
\text { EU/AU/NZ/SA only, } \\
\text { crowded spectrum, } \\
\text { large antenna, limited } \\
\text { bandwidth }\end{array}$ \\
\hline $\begin{array}{l}608-614 \\
1395-1400 \\
1427-1432\end{array}$ & WMTS & $\begin{array}{l}\text { Good propagation } \\
\text { characteristics, medical } \\
\text { only, antenna size } \\
\text { reasonable }\end{array}$ & $\begin{array}{l}\text { Licensed secondary use } \\
\text { limited to healthcare } \\
\text { providers inside } \\
\text { healthcare facilities in } \\
\text { US, limited spectrum, } \\
\text { heavily used }\end{array}$ \\
\hline $868-870$ & $\begin{array}{l}\text { General } \\
\text { Telemetry }\end{array}$ & $\begin{array}{l}\text { Good propagation } \\
\text { characteristics, } \\
\text { excellent building } \\
\text { penetration } \\
\text { characteristics }\end{array}$ & $\begin{array}{l}\text { EU only, limited } \\
\text { spectrum, heavily used }\end{array}$ \\
\hline $902-928$ & ISM & $\begin{array}{l}\text { Good propagation } \\
\text { characteristics }\end{array}$ & $\begin{array}{l}\text { US/Canada only, } \\
\text { crowded spectrum, } \\
\text { used by low powered } \\
\text { unlicensed devices, } \\
\text { harmful interference }\end{array}$ \\
\hline $\begin{array}{l}2400-2483.5 \\
2400-2500\end{array}$ & ISM & $\begin{array}{l}\text { Worldwide availability, } \\
\text { small antenna, large } \\
\text { bandwidth }\end{array}$ & $\begin{array}{l}\text { Crowded spectrum, } \\
\text { many standards and } \\
\text { technologies }\end{array}$ \\
\hline $5725-5850$ & ISM & $\begin{array}{l}\text { Worldwide availability, } \\
\text { small antenna, large } \\
\text { bandwidth }\end{array}$ & $\begin{array}{l}\text { Existing standards and } \\
\text { technologies, severe } \\
\text { attenuation }\end{array}$ \\
\hline $\begin{array}{l}4200-4800 \\
7250-8500\end{array}$ & UWB & $\begin{array}{l}\text { Worldwide availability, } \\
\text { short range, low power, } \\
\text { huge bandwidth, does } \\
\text { not interfere largely }\end{array}$ & $\begin{array}{l}\text { Coexistence with high } \\
\text { data rate multimedia } \\
\text { applications, severe } \\
\text { attenuation }\end{array}$ \\
\hline
\end{tabular}


who are authorized to use this band are physicians and trained technicians [64]. Other technologies include unlicensed ISM, Medical Implant Communications Service (MICS), and UWB. ISM band is usually utilized to protect adjacent channel interference, and additionally it is used by other classical Wireless Sensor Network (WSN) technologies. In this context UWB technology seems to be the most promising band candidate for the future generation WBANs.

In Table 1 we compare the main properties of the communication and data transmission bands in BAN and WBAN networks.

In this section we first define the main concept of UWB technology and then present possible benefits of the deployment of this mechanism in WBANs. We focus on the aspects of the whole system and transmitter design, communication channel modeling and the project of the physical layer of WBAN. The models are presented along with a short survey of the most notable and projects proposed in the literature.

\subsection{UWB Technology}

UWB RF technology allows a robust and energy effective data and signals transmission through wireless networks. By using a wide bandwidth, UWB offers an effective and low-cost combination of data and energy management systems by utilizing bands within the frequency range of 3.1-10.6 GHz. UWB signals have an inherent noise-like behavior due to their extremely low maximum effective isotropically radiated power. This makes them difficult to detect, which is crucial in the medical applications, where security aspects are very important. There are various implementations of UWB technology which differ in frequency band and signal characteristics. The most common UWB model is based on a specification of the multi-layer WiMedia Alliance protocol ${ }^{1}$ presented in Fig. 2.

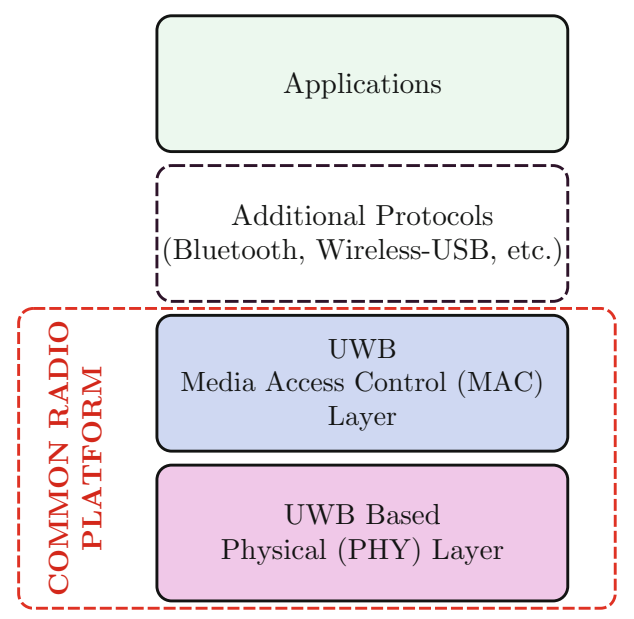

Fig. 2. WiMedia UWB protocol (see also [6])

${ }^{1}$ www.wimedia.org/en/index.asp. 
Two main layers of the WiMedia Alliance protocol are UWB based Physical (PHY) and Media Access Control (MAC) layer, which together form the "common radio platform" in the protocol stack. In PHY layer the frequency spectrum is divided into 14 bands and 6 band groups as shown in Fig. 3.

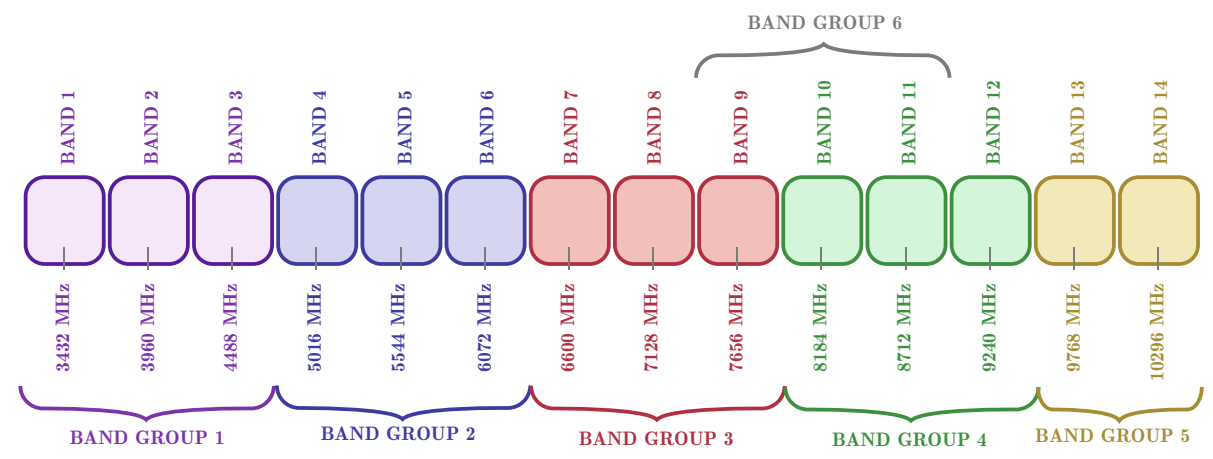

Fig. 3. UWB frequencies and band groups

Additional technical specifications for UWB include: (a) Multi-band Orthogonal Frequency Division Multiplexing with over 110 sub-carriers per channel (4.125 MHz bandwidth sub-carrier bandwidth), (b) a channel bandwidth of $528 \mathrm{MHz}$, and (c) a low broadcast power that allows same-channel coexistence with the other close band devices such as $802.11 \mathrm{a} / \mathrm{b} / \mathrm{g} / \mathrm{n}$ and Bluetooth radios [6]. The MAC protocol defines a multi-frame (Super-frame [6]) structure with sequentially executed beacon and data periods (see Fig. 4).

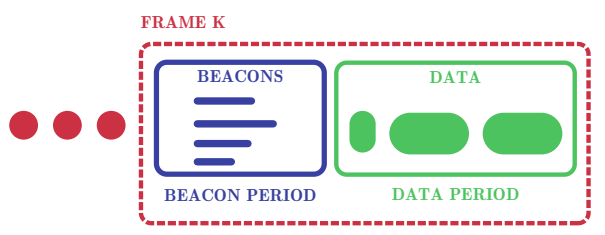

FRAME $\mathbf{K}+1$

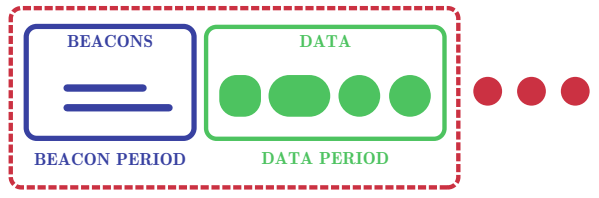

Fig. 4. UWB MAC frames

Beacons are transmitted by the UWB device(s) in order to provide the timing and resource access information, which is passed on and transmitted during the data phase. The PHY and MAC layers can be additionally combined with the other wireless protocols, such as Wireless-USB or Bluetooth 3.0, as an additional option for integration into mobile devices. 


\subsection{UWB BAN System Model}

There is no standardized architecture or system design for UWB BANs, but research is being done in this area and a number of works have been proposed with different designs for the architecture of BAN system. In [69], Roy et al. have proposed a promising architecture for body area networks, i.e., a multi-sensor multi-antenna architecture. In this approach, every sensor carries an antenna and the sink is placed in closed vicinity of a body. This sink is the central device and it supports an antenna array. Most of the computational complexity lies in the sink. Authors have proposed a complete analytical channel model for the onbody diffracted waves mechanism. IEEE 802.15.4a group has developed a low cost, low complexity, low power and low range physical layer based on UWB technology. The under discussion architecture is also built on the channel model of IEEE 802.15.4a. UWB is chosen because its low power spectral density and large bandwidth offers many advantages, such as low interference, low sensibility to fading, and accurate positioning. This architecture is expected to exploit the array gain and in turn increase the (Signal to Noise Ratio) SNR. Since power saving is a critical issue in such systems, the architecture minimizes the transmission power requirement and increases the battery lifetime of sensors. Different measurements were taken to test the proposed architecture in different scenarios. Issues like path loss, tapped delay time, and correlation aspects are discussed in detail. Tap mean amplitude decays with the delay and is described by a dual-slope power law. Parameters depend on the locations of transmitting and receiving antennas. This work only considers the first waves interfering with the human body. More research needs to be done on the delayed waves which are coming after reflection through the surroundings.

In [39], Kim et al. have proposed an UWB system employing binary zero correlation duration (ZCD) code for wireless BANs. As different devices are mounted on human body so there are chances of multiple access interference (MAI); in order to avoid this and to enhance the performance, binary ZCD is used as a spreading code for ultra wide band. This work shows that system performance can be enhanced considerably without adding much complexity by applying ZCD code.

In [68], Rajagopal et al. have proposed a chaotic based UWB system. The system meets the ultra low power requirements of body area networks while providing $100 \mathrm{kbps}$ to $10 \mathrm{Mbps}$ data rates, thus enabling a wide range of applications. Chaotic UWB modulation scheme is used to attain spectrum flexibility at minimum cost and power.

In [15], Chen et al. have explored the application of cooperative communications in UWB body area networks. Cooperative communications make use of available user terminals as relays that cooperate together to form a virtual antenna array. This work focuses on the system deployment scenario when the subject is in sitting posture. Vital channel parameters, such as path loss, power delay profile, power variation, and effective received power crosscorrelation, are investigated. The main feature of this work is that it utilizes the directional antennas in such a way so as to minimize human exposure to 
electromagnetic radiation keeping health concerns in mind. This work also provides a full-bandwidth view of a body-centric channel through a time-domain apparatus. The method used calculates the system diversity levels and it is expected to serve as a standard for assessing the performance of various diversity based techniques in body centric scenarios.

\subsection{UWB Transmitter Design for BANs}

The power saving requirements of BANs impose some constraints on the transceiver architecture and UWB signaling strategy. Implementation of UWB transceivers is still in the phase of research and conventional trends cannot be followed because of these constraints. So, the logical alternative is to look for analog front end implementations and simplest possible transceiver architectures. Low power UWB implementation for BANs have to meet the following design guidelines:

1. Low duty cycle signaling,

2. Non-coherent architecture at the receiver,

3. Pulse generator consuming low power, and

4. In order to comply with regulations, increase spreading factor in case of short pulse waveforms. That is, the average power concentrated in a pulse duration is spread into the spreading factor.

UWB-based systems use pulse position modulation (PPM) to transmit information with the $40 \mathrm{MHz}$ clock frequency of modulator. In [70], Ryckaert et al. highlight the potential of UWB for short-range communication. Most of the UWB transmitter building blocks are switched off between the pulses, which allows to minimize the energy consumption. Authors have evaluated the overall power consumption of the transmitter through a link budget analysis in a wireless BAN context, taking into account explicit channel models. A comparison of narrow-band implementations with UWB shows great reduction in power consumption. In [34], Kohno et al. have presented an ultra low power transceiver design for BANs. Transmitters and detectors are also described. System is analyzed in terms of packet error ratio, link budget, and power consumption. This can be done by adopting the IEEE 802.15.6 standard.

If research maintains its current momentum in this area, further enhancements are expected both at circuit and algorithmic level which will make UWB a promising and key technology for ultra-low-power communications.

\subsection{WBANs Antennas for UWB Frequencies}

Another important issue of WBANs is the antenna design which should meet the size limit and biocompatibility requirements. The antenna for a wireless body area network faces numerous RF challenges. Several antenna designs have been proposed in the literature, a few of them are discussed below.

In [84], Yazdandoost et al. have presented an antenna design which operates in UWB frequency from 3.1 to $5.1 \mathrm{GHz}$. Two layers of substrate are used in the 
antenna to cancel the effects of human body on performance of the antenna. Performance is then analyzed in free space as well as close to the human body. Experiments in different scenarios are performed without making any changes to the antenna design. The antennas become directional when placed close to the human body as it affects the radiation patterns of antenna. Similarly, human body absorbs a large amount of output power which affects performance. The proposed antenna design provides better performance when placed close to the human body due to the second substrate layer.

In [38], Attari et al. have presented a small size ultra wide band antenna for BANs. The proposed antenna works in the range of $3.1-10.6 \mathrm{GHz}$ in free space, which is then modified by using a PEC (perfect electric conducting) plane. PEC is used as a reflector to enhance performance when antenna is in close proximity to the human body. Simulation results show that the modified antenna has better performance in frequency and time domain. The proposed antenna design reduces the effect of the body on the antenna, thus, increasing the gain of antenna when placed close to the human body by using a PEC plane.

In [2], Almpanis et al. have presented an inverted truncated annular dielectric resonator antenna for BANs. The antenna operates at $3.4-5.0 \mathrm{GHz}$. The properties of antenna are discussed theoretically and practically. Performance is analyzed in free space and close to human body and demonstrates good results in frequency, as well as time domain. Although the design is presented specifically for BANs, the design concepts are general and it can be used for other applications with minor modifications.

\subsection{Using UWB for Transmission Channel Modeling in WBANs}

The high capacity transmission channel of the UWB systems is one of the most important advantage due to which UWB is employed in on-body and in-body medical monitoring systems. Research has been done on the study of path loss for various onbody and in-body devices. As data rate for BANs is very low and the range is limited, UWB is being considered as most suitable air-interface. A lot of work has been done on path loss model but the propagation channel has not been discussed in detail so far. When two sensors are communicating on the human body, a receiver can receive the signal in 3 ways: (a) propagating through the body, (b) diffracting around the body, and (c) reflections off nearby scatters and then back toward the body. The human body itself is a quite complex environment and no one has studied it explicitly from the perspective of wireless communication. Several channel models are presented in $[8,72,73,81]$.

In [72], Takizawa et al. have proposed stochastic channel models on power delay profile and path loss. All the models are derived from CTF (channel transfer functions) in a hospital room. Path loss model is presented for several bands while power delay profile is only presented for UWB.

In [73], Aoyagi et al. have provided channel models for BANs in UWB frequency band. The statistical model and parameters are extracted directly from measured channel CFTs. Channel models are derived on both path loss and power delay profile. In [8], Betancur et al. have proposed a similar statistical channel model. 


\subsection{UWB BAN Physical Layer}

According to the technical requirement of the WBAN task group, many companies and research institutes have proposed physical layer architectures to provide fundamental technologies for WBAN communication systems. Since there are various service scenarios for in-body or on-body applications, the physical layer proposals include UWB and narrowband techniques [14]. The physical layer is responsible for: (a) activation and deactivation of the radio transceiver, (b) Clear Channel Assessment (CCA) within the current channel, and (c) data transmission and reception.

In [17], Choi et al. have proposed the design of a PHY simulator for wireless BAN system in IEEE 802.15.6. As there are varying scenarios for body area networks, including in-body and on-body scenarios depending on the application, the physical layer proposals include UWB, as well as narrowband techniques. In the WBAN PHY case, the modulation method is adjusted according to frequency band and data rate. Several works on performance evaluation based on different channel models for UWB BANs are present in the literature. In [13], Chang et al. have investigated the performance of UWB-MIMO for BAN channel capacity through extensive measurements for both spatial and polar antenna arrays. Channel frequency responses were measured in $3-10 \mathrm{GHz}$ frequency range that covers the whole UWB band. Effects of bandwidth, array spacing, antenna polarization, and propagation conditions, from the measured channels, were analyzed on the UWB-MIMO channel capacity. MIMO channels are formed by combining a number of single input single output channel responses. It was observed that the MIMO channel capacity decreases with bandwidth and frequency; furthermore, the receiving power decreases when frequency band is increased. High frequency components have low power, so they don't contribute much to the channel capacity. Similarly, when the receiving array spacing is increased, the channel capacity and the spatial correlation co-efficient are decreased in most of cases, and the maximum value of power difference between any two sub-channels is increased. In order to achieve maximum channel capacity, array spacing of the spatial array should be lower than one wavelength. Device compactness can be achieved without sacrificing channel capacity in case of a polar array, in contrast to spatial arrays in non-line of sight (NLOS) conditions; in line of sight (LOS) conditions the maximum achievable capacity of a spatial array is higher than that of the polar array.

In [71], Sangodoyin et al. have investigate on the impact of body mass index (BMI) on the body-to-body propagation channels. They provided a detailed description of a measurement campaign for wireless body-to-body propagation channels. Measurements were done in an anechoic chamber to clearly work out the impact of the body. Channel frequency responses were measured in $2-10 \mathrm{GHz}$ frequency range.

In [73], Takizawa et al. have presented performance evaluation of UWB wireless BANs, which shows that the modulation scheme has to pay much penalty if it uses a non-coherent receiver as compared to coherent detection. In [23], Domenicali et al. have analyzed the performance of BAN composed of IEEE 
802.15.4a UWB sensors. The parameters used for performance evaluation are BER (bit error rate), network lifetime, and throughput. The work presents a unique case by analyzing performance in the presence of an external interfering network. BAN performance can be improved by using an optimized time hopping code assignment strategy.

In [80], and [22], performance evaluation method is presented which observes the waveform distribution along the signal path. Performance of a transceiver is evaluated in terms of BER. Results show that the human body casts more effect on performance than the environment, especially in case of NLOS path for a propagation channel. Selection of suitable pulse shape depends upon the modulation schemes used.

\subsection{Optimal Locations for Sensors in UWB BANs}

Knowledge of precise locations of sensors is necessary for successful data transmission in many medical applications. In WBAN these locations must be estimated with a very high (millimeter-scale) precision. Localization in WSNs has been actively studied during the last few years. Many methodologies effective in the standard wireless architectures, such as the received-signal-strengthindicator (RSSI) technique [35] and time-of-flight (TOF) measurement technique [10], can be successfully applied in WBANs and other medical applications.

In [1], Abbasi et al. have investigated the optimum locations for placing sensors on human body. Experiments were performed on more than 100 transceiver locations for LOS and NLOS scenarios. Path loss models were developed and performance was evaluated for different scenarios. An orthogonal frequency-domain multiplexing (OFDM) based UWB system was used and calculations were performed on the basis of BER values (see Table 2), on selected locations. Best possible locations identified by this work are legs and arms for LOS and NLOS scenarios, respectively.

Table 2. BER values for different parts of human body [1]

\begin{tabular}{l|l|l}
\hline \multirow{2}{*}{ Body part } & \multicolumn{2}{l}{ On-body } \\
\cline { 2 - 3 } & LOS (Front side) & NLOS (Back side) \\
\cline { 2 - 3 } & BER & BER \\
\hline Trunk $(\mathrm{Rx} 23 / \mathrm{Rx} 75)$ & $3.7 \mathrm{e}-2$ & $9.98 \mathrm{e}-4$ \\
\hline Left arm $(\mathrm{Rx} 27 / \mathrm{Rx} 90)$ & $3.89 \mathrm{e}-5$ & $5.1 \mathrm{e}-5$ \\
\hline Right arm $(\mathrm{Rx} 38 / \mathrm{Rx} 89)$ & $4.76 \mathrm{e}-3$ & $7.67 \mathrm{e}-5$ \\
\hline Left leg $(\mathrm{Rx} 44 / \mathrm{Rx} 104)$ & $4.83 \mathrm{e}-6$ & $2.34 \mathrm{e}-5$ \\
\hline Right leg $(\mathrm{Rx} 47 / \mathrm{Rx} 101)$ & $1.83 \mathrm{e}-5$ & $1.26 \mathrm{e}-4$ \\
\hline Head $(\mathrm{Rx} 54 / \mathrm{Rx} 57)$ & $9.99 \mathrm{e}-4$ & $6.54 \mathrm{e}-4$ \\
\hline
\end{tabular}


State-of-the-art of 3D localization systems have been surveyed in [42]. In [40] the authors present a methodology of the transmission of a 300 ps Gaussian pulse, which is modulated by an $8 \mathrm{GHz}$ carrier signal as a direct support to a localization system for indoor WBANs that provides accuracy in the $1-10 \mathrm{~mm}$ range and seems to be one of the most promising solutions for the future generation positioning techniques.

\section{Cloud Support to Mobile e-Health Systems and WBANs}

E-health system was considered as a solution for the presented model because it is the most powerful and it allows to interact remotely between patients and professionals. Due to providing data from WBAN to this system, these can help to solve many of the patient's medical problems. Although communication protocols and efficient data transmission between sensors, network body, local base node, and the external network base station (signal receivers) are crucial in the design of modern WBANs, data processing and replication, remote patient monitoring by medical personnel, and a fast and secure access to the data still remain challenging and complex issues in today's IT-based health care systems. Conventional medical systems are still built on workflows that consist of paper medical records, duplicated test results, handwritten notes. The U.S. Department of Health and Human Services has published in 2009 the Health Information Technology for Economic and Clinical Health (HITECH) Act [33] which contains a format known as an Electronic Medical Record (EMR) [44] for digitizing the patients' data and medical tests to make them transparent to patients together with health plan costs, covered services, and health insurance. A successful practical implementation of such U.S digitization idea (and its extension to be a global system) needs a low-cost scalable IT infrastructure that allows a fast remote access of patients and doctors to the system and data. This system must be easily adaptable to various patients' and other users' needs, departmental policies and organizational sizes, and security and data protection must be the paramount system's characteristics. Recently, cloud computing seems to be a good candidate for supporting IT-based health medical systems as it successfully offers multiple benefits for enterprise computing environments [67].

Cloud computing enables convenient and on-demand access to a shared configurable computing resources of various types, namely physical infrastructures (servers, datacenters, networks), virtual servers and web-based services to distributed users [31]. All these resources are provisioned by the service providers and local resource providers with possible minimal cost and management efforts and interactions. Major characteristics of cloud computing which are important in medical data storage, data analysis and processing, can be defined as follows $[11,46]$ : 
- on-demand self-service: each cloud user can deploy and configure the cloud servers and services himself, no interactions with service providers are necessary;

- multitenancy: the resources and costs are shared across a large community of cloud users;

- scalability: an efficient and low-cost configuration and assignment of system resources according to consumer demand;

- easy system access: cloud resources and services are accessed through standard (Internet) protocols, using standard web browsers regardless of their location and platform, e.g., smart phones.

- meeting security demand: dedicated services for higher security requirements, [76].

Model described above is supported by cloud-based architecture. Data from body sensors in WBANs are received by Cloud Client and then, they are sent directly to the cloud, which consist with the providers of the service such as: Software as a Service (SaaS) that is a cloud computing model in which the application is running on the service provider's computers and it is available to users via the Internet; Platform as a Service (PaaS) which provides the runtime environment and the system on which the application runs; Infrastructure as a Service (Iaas) which provides the appropriate equipment to implement the described model and allows to its scalability at any time [30]. These services collect received from Cloud Client data from sensors in a Data center with access to Cloud Storage Facilities. The general architecture of cloud-based WBAN support infrastructure is presented in Fig. 5 .

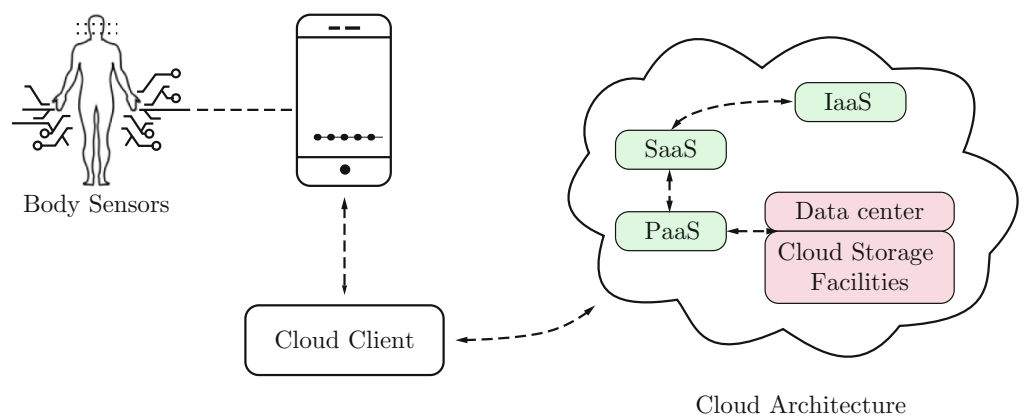

Fig. 5. Cloud-based support to WBANs

In this model a patient, equipped with BAN sensors and a mobile device, such as smart phone or a tablet, communicates to the cloud system through a cloud web client or a specially designed standalone application. The base sensor module in WBAN collects the patient's data and transmits it to the mobile device (often via Bluetooth) without the patient's intervention. The cloud client (cloud platform interface), installed at the mobile device, forwards the patient's 
data to the appropriate web service installed at the cloud platform. The collected data is stored, analyzed, and processed by a software module. The results of this analysis (and possible diagnosis) are disseminated to the patient, his doctor, who can be another mobile cloud user, emergency services (if necessary), hospital etc., at specified time intervals or just single time period (point). Patient's historical medical reports are available for retrieval from the cloud after user authentication.

Although many popular cloud computing platforms, either free (e.g., iCloud [36] and DropBox [25]) or commercial (e.g., GoGrid [28], Amazon AWS [4], Google Cloud Platform [29]) are already available for pervasive management of large volumes of user's data, they may not be suitable for many health care applications. Moreover, cloud computing for health care needs highest level of availability, security, and privacy for the patients' data in order to gain acceptance in the marketplace. Development of high security and accessibility of cloud computing services and personalization of processed patients' data are contentious issues in the design of scalable IT-based systems supporting WBANs [37].

\subsection{State-of-the Art}

The integration of WBANs and cloud service providers to provide mobile health facilities has transformed from a vision of future to a nowday facility. Numerous renowned hospitals in Europe and US provide e-health services based on Electronic Health Records (EHR) with cloud integration [58]. In the following lines we discuss the state-of-the art research proposals regarding ubiquitous health based on WBAN-cloud integration.

In [46] the authors present the could-based support for the analysis of the electrocardiogram (ECG) data. The proposed system model consist of the three traditional cloud layers, that is: (a) Software, (b) Platform, and (c) Infrastructure. The software layer provides data upload, storage, and analysis facilities. The platform layer consists of three modules: (a) container scaling manager, (b) workflow engine [45], and (c) Aneka scheduling environment [79]. The workflow engine is installed inside the container and manages the execution of the workflow process in ECG analysis. The ECG data processed in this workflow can be either numerical or graphical (ECG) [7], and are calculated and compared with the patients historical data and standard values. The container scaling manager creates new containers according to the number of incoming requests (tasks). The tasks composed in the workflow module are submitted to the Aneka [79] scheduling environment. This scheduling system plays the role of a workflow distribution and management platform based on the conventional master-slave model adopted in cloud systems. The Aneka master is installed on the platform cloud layer while the slaves are task executors installed on the infrastructure entities (virtual machines) of the cloud. For the security of data communication between different layers, a third party Public Key Infrastructure (PKI) is proposed. This model is a good example of the utilization of the public cloud environment for real-time monitoring the patient's health condition. 
Similar idea is presented as the HealthCloud project in [24]. The cloud service client is designed as a web application for the Android OS consisting of: (a) Patient Health Record module which retrieves patient records form the cloud, (b) Medical Imaging module that decodes and displays the image according to the Digital Imaging and Communications in Medicine (DICOM) [19] and JPEG2000 compression standard. The Amazon's S3 cloud service [4] has been utilized for the evaluation of the developed model in WLAN and 3G networks with different JPEG2000 compression parameters. SSL data encryption is proposed to secure data communication. A similar model based on Internet of Things (IoT) communication framework for mobile health care is described in [48].

Doherty et al. [60] have proposed a physiological lifelogged data framework that stores lifetime events of a person over a cloud. While the lifelogged data can help people remember what they were doing at a particular moment, it can also be analyzed to find out potential reasons behind a medical condition. The proposed 'SmartLogger' system provides three interfaces to view the physiological data. The query interface allows a user to search data based on temporal queries. The chart interface provides graphical images of physiological activities, such as the heart rate measured by a ECG sensor. The context interface provides additional information gathered from various bio-sensors such as the location of the user, images of the surroundings, and audio data that might be helpful in classifying a temporal event.

Research has also been done on cloud-sensor integration to build communitycentric information sensing and dispensing applications that work on the basis of publication subscription model [50]. A publication-subscription broker that delivers information to users via a cloud service application consists of: (a) a sensor data stream monitoring and processing component, (b) a registry component that saves information about user subscriptions to different applications, (c) an analyzer component that maps data streams to corresponding applications, and (d) a disseminator component that sends data to a subscribed user using an event matching algorithm.

WBAN-cloud integration for mobile health care systems is a novel idea with benefits of computational and storage offloading. However, researchers have opposed cloud computing approach to ubiquitous healthcare due to the communicational overhead such a system incurs, delays experienced, and continuous connectivity requirement with the cloud [49]. Instead, a ubiquitous healthcare system based on a resource provisioning framework that harnesses the computational and storage capabilities of computing devices lying in close vicinity is proposed. The authors of [49] have proposed a wireless communication model to transmit patient data and to prioritize data streams based on severity of patient's condition. The model is a two-tier hierarchical architecture consisting of inter and intra-BAN communications. Inside each BAN, a cluster head $(\mathrm{CH})$ is selected based on higher computational and communicational power to act as a gateway for all inter-BAN sensor nodes. The $\mathrm{CH}$ may himself act as a broker or offload the tasks to a base station. The broker of the proposed system is 
composed of a workflow manager and a scheduler/optimizer. The workflow manager receives service requests from sensors. The optimizer identifies the appropriate service providers in the vicinity for service distribution. The service provides volunteer themselves or they are identified by request-response messages. These messages contain the computational (CPU cycles) and storage (bytes) facility available at a service provider. In this manner ubiquitous health can be provided without the requirement of cloud connectivity.

\section{$5 \quad$ Security Challenges and Solutions}

BANs are meant to support medical applications mainly. Hence, privacy, security, (Quality of Service) QoS, and reliability are important factors besides energy efficiency. Traditional security and privacy techniques are not appropriate for BANs due to bounded processing power, memory, and energy, as well as the lack of a user interface, unskilled users and global roaming. Hence, novel lightweight and resource-efficient methods have to be developed for BANs [66]. Global roaming over heterogeneous infrastructure networks further complicates end-to-end security provisions. Cloud integration of mobile health applications has given rise to new security concerns such as trustworthiness of the cloud client and secure extra-BAN communication.

Security and privacy of patients' data when it is stored in and retrieved from the cloud, user authentication and authorization for access to the data, and internal management of data by the cloud provider are major concerns in the cloud-based mobile health applications. Researchers have proposed the use of TSL/SSL protocol to secure communication between the cloud and the patient $[24,59]$. The trust between the cloud and the patient is still a major research issue to be dwelled upon. The process of authentication between a user and a service provider for the identification of proper authorization level is based on trusted third party certification authority in many cloud based applications. The trusted third party certification authority is responsible for the implementation of the authentication and authorization system based on the PKI. Cloud support provides the opportunity to offload computationally intensive jobs form the mobile devices to the cloud. In the following lines we present state-of-the art research proposals for security challenges in WBAN and WBAN cloud integration.

Nkosi and Mekuria [43] propose a security service provision framework based on the cloud services as a solution for mobile health concerns. Security is provided as a service to the mobile devices like other services provided by the cloud. In this model, the mobile devices are cloned over the cloud infrastructure. When the mobile device faces a computationally intensive task, it transfers the task to its clone over the cloud. The task is executed in the cloud and the output is sent back to the mobile device. The only concern about Security As a Service (SasS) model is the security of the communication channel between the cloud and the mobile device. This threat is mitigated by implementation of secure communication protocols. Similar task off-loading scheme has been presented in [55]. Task 
offloading techniques help the resource constrained mobile devices and sensors to execute their task over the cloud, but they also incur communication overhead.

Wang et al. [57] have proposed a distributed dynamic integrity check and data storage scheme for WSNs. The data to be encrypted is divided into $n$ blocks. Each data block is encrypted using a secret key. Each encrypted data block is distributed among $n$ neighbors, thus providing secure data storage. When an integrity check is required for the data, each node having a share of the $n$ blocks of the data computes and broadcasts its integrity checksum. Thus, the distributed storage and computation reduces the memory overhead on a single sensor node.

To secure medical data from a determined adversary, Pietro et al. [62] have proposed a data survivability scheme based on continuous movement of data from one sensor node to another. It is assumed that the adversary knows the origins of the target data and can compromise a subset of nodes to capture that data. The continuous data communication, replication, and encryption applied in this scheme makes it impractical for energy constrained WBANs.

Access privileges to medical data in WBANs can be maintained by implementing a Role-Based Access Control (RBAC) model [65]. The participating WBAN entities are assigned groups according to their roles, such as physicians and patients. Each user role is mapped to user-privilege set in one to many mappings. RBAC is simplistic and well suited for the requirements of WBANs. An RBAC scheme based on secret key encryption for medical WBANs is proposed in [63]. The proposed scheme utilizes the random key predistribution methods defined in [53]. By predistributing encryption keys, a user can establish a pairwise keys with all entities in the WBAN. Each pairwise key is mapped to a role, thus, access privileges are assigned to each pair of keys. The predistribution and pairwise key management make this scheme impractical for large WBANs configurations.

In [74] a secure data communication model for scalable cloud computing applications is presented. The model adds a Security Manager entity to the generic cloud model. The security manager is a trusted third extension of the cloud provider. The security manager generates public and private keys of each user, regenerates keys after expiry, generates session keys and maintains access control list for each data set.

A data re-encryption scheme proposed in [52] is used to offer confidential data storage that is even private to a cloud provider. A user encrypts the data before sending it to the cloud where the security manager re-encrypts the data before it is stored in the cloud. The authors have also provided a variation of the model in which data encryption is offloaded to the security manger while data re-encryption is performed by the cloud provider.

In [56] researchers have presented a novel cross-cloud domain based trust model. A trust value is associated with each client and domain entity. When an entity A wants to communicate with an entity B, it compares the entity B's trust value with its trust threshold. If the trust value is greater than the trust threshold, it will continue with the transaction. Otherwise it will abort 
the operation. Trust values are updated on the basis of completed transactions which have not violated trust relationship between two entities.

Sorber et al. [61] have proposed a novel mHealth model based on a wristwatchlike wearable device, Amulet. An Amulet provides many advantages over mobile and PDA based mHealth modes such as: (a) Amulet is tightly coupled with the patient's body unlike mobile devices and PDA's, which can be out of transmission range of the sensor devices, (b) Amulet uses special-purpose hardware to support secure mHealth applications. Unlike mobile devices, the Amulet provides secure storage of encryption keys, (c) the Amulet provides interoperability between sensor and mobile devices by implementing multiple low-power radios supporting diverse wireless technologies, and (d) Amulet is able to authenticate the wearer by various physiological parameters, such as pulse and galvanic skin response (GSR).

Researchers [51] have proposed a Plug-n-Test approach to secure medical data sensing and processing in mobile phones based on plug-in smart cards that provide a trusted computing platform. The smart card provides processing and storage of encrypted data. The smart card thus exchanges keys with medical sensors and the back-end cloud for sending and receiving encrypted data. The test model includes a JavaCard applet and two Android phones with smatcard integration. The test model implementation shows $20 \%$ energy consumption overhead in worst case, $59-69 \%$ increase in latency time, and $44 \%$ communication overhead while ensuring data confidentiality and integrity.

The proposals discussed in the above section ensure data security which is critical in the context of medical health records but each proposal also incurs computational and communicational overheads while ensuring data security. A lightweight, universally accepted security framework for WBANs providing ubiquitous health facilities is an area with great research potential.

\section{Conclusions and Future Directions}

The WBAN is an emerging and promising technology that is expected to change people's daily life and healthcare experiences. Data security, safety, efficiency, and privacy in WBANs are the key research issues, and a number of considerable challenges still remain to overcome. The research in this area is still in its infancy, but it is believed it will draw a huge amount of interest in next few years.

This chapter has highlighted many research and practical issues related to WBANs supported by the UWB wireless technologies. UWB wireless technology for intra-BAN communications is one of the most promising methodology for the effective data transmission in today's medical applications. However, some early results on the application of the other technologies, such as Bluetooth and ZigBee, are also available, but no standard has been developed so far for WBANs.

There is a big potential in designing a physical layer for WBANs. Previous work includes some UWB and narrow band techniques. A channel modeling field is also in a research phase and the complex environment of the human 
body poses many challenges to researchers in this regard. There is no standard antenna design for wireless BAN devices. Antenna needs to be comfortable and compatible with the human body. The issues of directionality, antenna gain needs to be investigated in detail when working around the human body. Neither a transmitter design has not been finalized yet. Different transmitter designs have been proposed with the focus on low-power transmitters. Apart from these technical details, there are a lot more issues to be resolved for social acceptance of BANs. These include conflicts between different requirements, such as security and efficiency.

Cloud computing infrastructure provides a cost effective and easy to use data sharing and access platform which is ideal for the IT enabled healthcare systems. Requirements of such a cloud supported health system is high availability of data for healthcare personnel and security of the patients' data. Authentication and authorization solutions provided by third parties have been proposed as a solution for data confidentiality requirements.

Catering all these challenges is most likely to require new methods and protocols for MAC and PHY, and state-of-the-art antenna design, transmitter design, and lightweight security protocols. UWB is being considered as most promising candidate for BANs. The state-of-the-art in system design, channel modeling, performance evaluation, transmitter design, and antenna design of UWB BANs is also presented in this chapter. The WBAN-cloud integration and related security challenges have also been thoroughly surveyed. Researchers, engineers, and practitioners from various disciplines, must come together to overcome technical roadblocks in order to bring the vision of a ubiquitous body area network to reality.

\section{References}

1. Abbasi, Q.H., Khan, M.M., Alomainy, A., Hao, Y.: Characterization and modelling of ultra wideband radio links for optimum performance of body area network in health care applications. In: 2011 International Workshop on Antenna Technology (iWAT), pp. 206-209 (2011)

2. Almpanis, G., Fumeaux, C., Fröhlich, J., Vahldieck, R.: A truncated conical dielectric resonator antenna for body-area network applications. IEEE Antennas Wirel. Propag. Lett. 8, 279-282 (2009)

3. Drude, S.: Requirements and application scenarios for body area networks. In: Mobile and Wireless Communications Summit (2008)

4. Amazon Web Services. aws.amazon.com

5. IEEE 802.15. www.ieee802.org/15/pub/TG6.html

6. Ayar, E.: UWB Wireless Video Transmission Technology in Medical Applications. NDS Surgical Imaging (NDSsi) White Paper-Report (2010)

7. Bahoura, M., Hassani, M., Hubin, M.: DSP implementation of wavelet transform for real time ECG wave forms detection and heart rate analysis. Comput. Methods Programs Biomed. 52(1), 35-44 (1997)

8. Betancur, L., Cardona, N., Navarro, A., Traver, L.: A statistical channel model for on body area networks in ultra wide band communications. In: Proceedings of the IEEE Radio and Wireless Symposium, vol. 1, pp. 715-718 (2008) 
9. Basu, S., Sarkar, M., Nagaraj, S., Chinara, S.: A survey on ultra wideband and ultrasonic communication for body area networks. Int. J. Ultra Wideband Commun. Syst. 3(3), 143-154 (2016)

10. Baunach, M., Kolla, R., Mvihlberger, C.: Beyond theory: development of a real world localization application as low power WSN. In: Proceedings of IEEE Conference on Local Computer Networks, Dublin, Ireland, 15-18 October 2007, pp. 872-884 (2007)

11. Buyya, R., Broberg, J., Goscinski, A. (eds.): Cloud Computing: Principles and Paradigms. Wiley, Hoboken (2011)

12. Cao, H., Leung, V., Chow, C., Chan, H.: Enabling technologies for wireless body area networks: a survey and outlook. IEEE Commun. Mag. 47(12), 84-93 (2009)

13. Chang, W.J., Tarng, J.-H., Peng, S.-Y.: Frequency-space-polarization on UWB MIMO performance for body area network applications. IEEE Antennas Wirel. Propag. Lett. 7, 577-580 (2008)

14. Cwalina, K., Sławomir, A., Rajchowski, P.: An off-body narrowband and ultra-wide band channel model for body area networks in a ferry environment. In: Proceedings of the IRACON 7th MC Meeting and 7th Technical Meeting, pp. 1-20 (2018)

15. Teo, Y.J., et al.: Cooperative communications in ultra-wideband wireless body area networks: channel modeling and system diversity analysis. IEEE J. Sel. Areas Commun. 27(1), 5-16 (2009)

16. Hoyt, R., Reifman, J., Coster, T., Buller, M.: Combat medical informatics: present and future. In: Proceedings of the AMIA 2002 Annual Symposium, San Antonio, TX, pp. 335-339 (2002)

17. Choi, B.K., Kim, B.S., Lee, S., Wang, K.Y., Kim, Y.J., Chung, D.: Narrowband physical layer design for WBAN system. In: Proceedings of the First International Conference on Pervasive Computing, Signal Processing and Applications, pp. 154$157(2010)$

18. Contaldo, M., Banerjee, B., Ruffieux, D., Chabloz, J., Le Roux, E., Enz, C.C.: A 2.4-GHz BAW-based transceiver for wireless body area networks. IEEE Trans. Biomed. Circuits Syst. 4(6), 391-399 (2010)

19. The Digital Imaging and Communications in Medicine (DICOM) Standard. medical.nema.org

20. Mare, S., Sorber, J., Shin, M., Cornelius, C., Kotz, D.: Adaptive security and privacy for mHealth sensing. In: Proceedings of the 2nd USENIX Conference on Health Security and Privacy (HealthSec 2011), pp. 2-12. USENIX Association, Berkeley (2011)

21. Merli, F., Skrivervik, A.K.: Design and measurement considerations for implantable antennas for telemetry applications. In: Proceedings of 4th European Conference on Antennas and Propagation EuCAP, pp. 1-5 (2010)

22. De Santis, V., Feliziani, M., Maradei, F.: Safety assessment of UWB radio systems for body area network by the FD2TD method. IEEE Trans. Magn. 46(8), 32453248 (2010)

23. Domenicali, D., De Nardis, L., Di Benedetto, M.-G.: UWB body area network coexistence by interference mitigation. In: Proceedings of IEEE International Conference on Ultra-Wideband, ICUWB 2009, Vancouver, Canada, 9-11 September 2009, pp. 713-717 (2009)

24. Doukas, C., Pliakas, T., Maglogiannis, I.: Mobile healthcare information management utilizing cloud computing and android OS. In: Proceedings of the 32nd Annual International Conference of the IEEE EMBS Buenos Aires, Argentina, 31 August-4 September 2010, pp. 1037-1040 (2010) 
25. DropBox. www.dropbox.com

26. Drude, S.: Requirements and application scenarios for body area networks. In: 16th IST Mobile And Wireless Communications Summit, pp. 1-5 (2008)

27. Gerrits, J.F.M., Farserotu, J.R., Long, J.R.: Low-complexity Ultra-Wide-Band communications. IEEE Trans. Circuits Syst. 55(4), 329-333 (2008)

28. GoGrid Storage Services. www.gogrid.com

29. Google Cloud Platform. cloud.google.com

30. Grzonka, D.: The analysis of OpenStack cloud computing platform: features and performance. J. Telecommun. Inf. Technol. 3, 52-57 (2015)

31. Grzonka, D., Szczygiel, M., Bernasiewicz, A., Wilczynski, A., Liszka, M.: Short analysis of implementation and resource utilization for the OpenStack cloud computing platform. In: Proceedings of 29th European Conference on Modelling and Simulation, ECMS 2015, pp. 608-614 (2015)

32. Hanson, M.A., et al.: Body area sensor networks: challenges and opportunities. Computer 42(1), 58-65 (2009)

33. HITECH. www.hhs.gov/ocr/privacy/hipaa/administrative/enforcementrule/ hitechenforcementifr.html

34. Hernandez, M., Kohno, R.: Ultra low power UWB transceiver design for body area networks. In: 2nd International Symposium on Applied Sciences in Biomedical and Communication Technologies, Israel 2009, pp. 1-4 (2009)

35. Hjortland, H.A., Lande, T.S.: CTBV integrated impulse radio design for biomedical applications. IEEE Trans. Biomed. Circuits Syst. 3(2), 79-88 (2009)

36. iCloud. www.icloud

37. Val, J., et al.: Mobihealth: mobile health services based on body area networks. In: Istepanian, R.S.H., Laxminarayan, S., Pattichis, C.S. (eds.) M-Health. Topics in Biomedical Engineering, pp. 219-236. Springer, Boston (2006). https://doi.org/ 10.1007/0-387-26559-7_16

38. Karimabadi, S.S., Attari, A.R.: Gain enhancement of small size UWB antenna for wireless body area network applications. In: IEEE Proceedings of ICEE 2010, 11-13 May 2010, pp. 50-53 (2010)

39. Kim, E.C., Park, S., Cha, J.S., Kim, J.Y.: Improved performance of UWB system for wireless body area networks. IEEE Trans. Consum. Electron. 56(3), 1373-1379 (2010)

40. Kuhn, M., Zhang, C., Mahfouz, M., Fathy, A.E.: Real-time UWB indoor positioning system with millimeter 3-D dynamic accuracy. In: Proceedings of IEEE Antennas and Propagation Society International Symposium, Charleston, CS, 1-5 June 2009 (2009)

41. Latré, B., Braem, B., Moerman, I., Blondia, C., Demeester, P.: A survey on wireless body area networks. Wireless Netw. 17(1), 1-18 (2011)

42. Meier, C., Terzis, A., Lindenmeier, S.: A robust 3D high precision radio location system. In: Proceedings of IEEE/MTT-S International Microwave Symposium, Honolulu, HI, 3-8 June 2007, pp. 397-400 (2007)

43. Nkosi, M.T., Mekuria, F.: Cloud computing for enhanced mobile health applications. In: Proceedings of the IEEE 2nd International Conference on Cloud Computing Technology and Science, CloudCom 2010, pp. 629-633 (2010)

44. Open Clinical Knowledge Managemnt for Medical Care. www.openclinical.org/ emr.html

45. Pandey, S., Voorsluys, W., Rahman, M., Buyya, R., Dobson, J., Chiu, K.: A grid workflow environment for brain imaging analysis on distributed systems. Concurr. Comput. Pract. Exp. 21(16), 2118-2139 (2009) 
46. Pandey, S., Voorsluys, W., Niu, S., Khandoker, A., Buyya, R.: An autonomic cloud environment for hosting ECG data analysis services. Future Gener. Comput. Syst. 20, 147-154 (2012)

47. Chen, M., Gonzalez, S., Vasilakos, A., Cao, H., Leung, V.C.: Body area networks: a survey. Mob. Netw. Appl. 16(2), 171-193 (2011)

48. Bui, N., Zorzi, M.: Health care applications: a solution based on the Internet of Things. In: Proceedings of the 4th International Symposium on Applied Sciences in Biomedical and Communication Technologies (ISABEL 2011). ACM, New York (2011). Article 131, $5 \mathrm{p}$

49. Viswanathan, H., Chen, B., Pompili, D.: Research challenges in computation, communication, and context awareness for ubiquitous healthcare. IEEE Commun. Mag. 50(5), 92-99 (2012)

50. Hassan, M.M., Song, B., Huh, E.-N.: A framework of sensor-cloud integration opportunities and challenges. In: Proceedings of the 3rd International Conference on Ubiquitous Information Management and Communication (ICUIMC 2009), pp. 618-626. ACM, New York (2009)

51. Sorber, J.M., Shin, M., Peterson, R., Kotz, D.: Plug-n-trust: practical trusted sensing for mhealth. In: Proceedings of the 10th International Conference on Mobile Systems, Applications, and Services (MobiSys 2012), pp. 309-322. ACM, New York (2012)

52. Ateniese, G., Fu, K., Green, M., Hohenberger, S.: Improved proxy re-encryption schemes with applications to secure distributed storage. ACM Trans. Inf. Syst. Secur. 9, 1-30 (2006)

53. Wenliang, D., Deng, J., Han, Y.S., Varshney, P.K., Katz, J., Khalili, A.: A pairwise key predistribution scheme for wireless sensor networks. ACM Trans. Inf. Syst. Secur. 8, 2 (2005)

54. Li, M., Lou, W., Ren, K.: Data security and privacy in wireless body area networks. Wireless Commun. 17, 1 (2010)

55. Chun, B.-G., Maniatis, P.: Augmented smartphone applications through clone cloud execution. In: Proceedings of the 12th Conference on Hot Topics in Operating Systems (HotOS 2009), p. 8-8. USENIX Association, Berkeley (2009)

56. Li, W., Ping, L.: Trust model to enhance security and interoperability of cloud environment. In: Jaatun, M.G., Zhao, G., Rong, C. (eds.) CloudCom 2009. LNCS, vol. 5931, pp. 69-79. Springer, Heidelberg (2009). https://doi.org/10.1007/978-3642-10665-1_7

57. Wang, Q., et al.: Dependable and secure sensor data storage with dynamic integrity assurance. In: Proceedings of the IEEE INFOCOM, pp. 954-962 (2009)

58. Weiss, A.: Health and biomedical informatics. netWorker 13(4), 18-25 (2009)

59. Kurschl, W., Beer, W.: Combining cloud computing and wireless sensor networks. In: Proceedings of the 11th International Conference on Information Integration and Web-based Applications and Services (iiWAS 2009), pp. 512-518. ACM, New York (2009)

60. Doherty, A.R., Tolle, K.M., Smeaton, A.F.: Utilising contextual memory retrieval cues and the ubiquity of the cell phone to review lifelogged physiological activities. In: Proceedings of the 1st International Workshop on Interactive Multimedia for Consumer Electronics (IMCE 2009), pp. 19-26. ACM, New York (2009)

61. Sorber, J., et al.: An amulet for trustworthy wearable mHealth. In: Proceedings of the Twelfth Workshop on Mobile Computing Systems and Applications (HotMobile 2012). ACM, New York (2012). Article 7 
62. Di Pietro, R., et al.: Catch me (if you can): data survival in unattended sensor networks. In: 2008 Sixth Annual IEEE International Conference on Pervasive Computing and Communications (PerCom), pp. 185-194 (2008)

63. Morchon, O.G., Baldus, H.: Efficient distributed security for wireless medical sensor networks. In: 2008 International Conference on Intelligent Sensors, Sensor Networks and Information Processing, pp. 249-254 (2008)

64. Istepanian, R.S.H., Laxminarayan, S., Pattichis, C.S.: M-Health: Emerging Mobile Health Systems. Springer, New York (2005). https://doi.org/10.1007/b137697

65. Venkatasubramanian, K.K., Gupta, S.K.S.: Security solutions for pervasive healthcare. In: Xiao, Y. (ed.) Security in Distributed Grid Mobile and Pervasive Computing, pp. 443-464. Auerbach, Boston (2007)

66. Patel, M., Wang, J.: Applications, challenges, and prospective in emerging body area networking technologies. IEEE Wirel. Commun. 17(1), 80-88 (2010)

67. Qiu, X., et al.: Cloud technologies for bioinformatics applications. In: Proceedings of the 2nd Workshop on Many-Task Computing on Grids and Supercomputers, pp. 1-10. ACM (2009)

68. Rajagopal, S., Kang, N.-G., Park, S.-H., Bynam, K., Cho, C., Won, E.T.: Chaotic UWB based system design for ultra low power body area networks. IEEE Trans. Microw. Theory Tech. 56(10), 15-18 (2008)

69. Roy, S., Oestges, C., Horlin, F., De Doncker, P.: A comprehensive channel model for UWB multisensor multiantenna body area networks. IEEE Trans. Antennas Propag. 58(1), 163-170 (2010)

70. Ryckaert, J., et al.: Ultra-WideBand transmitter for wireless body area networks. IEEE Trans. Circuits Syst. I 52(12), 2515-2525 (2005)

71. Sangodoyin, S., Molisch, A.F.: Experimental investigation of the impact of BMI on ultrawideband MIMO body-to-body networks. In: Proceedings of the IEEE 87th Vehicular Technology Conference (VTC Spring), pp. 1-5 (2018)

72. Takizawa, K., et al.: Channel models for wireless body area networks. In: Proceedings of the 30th Annual Conference of IEEE Engineering in Medicine and Biology Society, pp. 1549-1552 (2008)

73. Takizawa, K., Aoyagi, T., Kohno, R.: Channel modeling and performance evaluation of UWB-based wireless body area networks. In: IEEE ICC 2009 (2009)

74. Tysowski, P.K., Hasan, M.A.: Re-encryption-based key management towards secure and scalable mobile applications in clouds. In: IACR Cryptology ePrint Archive, pp. 668-668 (2011)

75. Lorincz, K., et al.: Mercury: a wearable sensor network platform for high-fidelity motion analysis. In: Proceedings of the 7th ACM Conference on Embedded Networked Sensor Systems (SenSys 2009), pp. 183-196. ACM, New York (2009)

76. Jakóbik, A., Grzonka, D., Palmieri, F.: Non-deterministic security driven meta scheduler for distributed cloud organizations. Simul. Model. Pract. Theory 76, 67-81 (2017)

77. Varshney, U.: Pervasive healthcare and wireless health monitoring. Mob. Netw. Appl. 12, 113-127 (2007)

78. Jakóbik, A.: Big data security. In: Pop, F., Kołodziej, J., Di Martino, B. (eds.) Resource Management for Big Data Platforms: Algorithms, Modelling, and HighPerformance Computing Techniques. CCN, pp. 241-261. Springer, Cham (2016). https://doi.org/10.1007/978-3-319-44881-7_12

79. Vecchiola, C., Chu, X., Buyya, R.: Aneka: a software platform for .NET-based could computing. In: High Speed and Large Scale Scientific Computing, pp. 267295. IOS Press (2009) 
80. Wang, S., Park, J.-T.: Modeling and analysis of multi-type failures in wireless body area networks with semi-Markov model. IEEE Commun. Lett. 14(1), 6-8 (2010)

81. Xia, L., Redfield, S., Chiang, P.: Experimental characterization of a UWB channel for body area networks. EURASIP J. Wirel. Commun. Netw. 2011, Article ID 703239, 11 p. (2011). https://doi.org/10.1155/2011/703239

82. Yan, S., Soh, P.J., Vandenbosch, G.A.E.: Wearable ultrawideband technology-a review of ultrawideband antennas, propagation channels, and applications in wireless body area networks. IEEE Access 6, 42177-42185 (2018)

83. Yang, G.-Z. (ed.): Body Sensor Networks. Springer, London (2006). https://doi. org/10.1007/1-84628-484-8

84. Yazdandoost, K.Y., Kohno, R.: UWB antenna for wireless body area network. In: Proceedings of the Asia-Pacific Microwave Conference, APMC 2006, pp. 1647-1652 (2006)

85. Zasowski, T., Wittneben, A.: Performance of UWB receivers with partial CSI using a simple body area network channel model. IEEE J. Sel. Areas Commun. 27(1), $10(2009)$

Open Access This chapter is licensed under the terms of the Creative Commons Attribution 4.0 International License (http://creativecommons.org/licenses/by/4.0/), which permits use, sharing, adaptation, distribution and reproduction in any medium or format, as long as you give appropriate credit to the original author(s) and the source, provide a link to the Creative Commons license and indicate if changes were made.

The images or other third party material in this chapter are included in the chapter's Creative Commons license, unless indicated otherwise in a credit line to the material. If material is not included in the chapter's Creative Commons license and your intended use is not permitted by statutory regulation or exceeds the permitted use, you will need to obtain permission directly from the copyright holder.

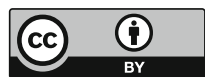

